\title{
Editorial
}

\section{Surgical Margins in Breast Conservation}

\author{
Sheldon Marc Feldman \\ Division of Breast Surgery, Vivian L. Milstein Associate Professor of Clinical Surgery, College of Physicians and Surgeons, \\ Columbia University, 161 Fort Washington Avenue, 10th Floor, Suite 1005, NY 10032, USA \\ Correspondence should be addressed to Sheldon Marc Feldman; sf2388@columbia.edu
}

Received 27 December 2012; Accepted 27 December 2012

Copyright (C) 2013 Sheldon Marc Feldman. This is an open access article distributed under the Creative Commons Attribution License, which permits unrestricted use, distribution, and reproduction in any medium, provided the original work is properly cited.

Significant progress has been made in the diagnosis and treatment of breast cancer during the past 30 years. The increased availability of screening mammography has resulted in a higher percentage of woman being diagnosed with early stage disease allowing the option of breast conservation therapy to be more widely available. Long-term follow-up studies clearly demonstrate equivalent survival with breast conservation surgery (lumpectomy) and radiotherapy versus total mastectomy $[1,2,3]$. The importance of obtaining clear lumpectomy surgical margins has been well established in minimizing the risk of local recurrence [4]. Unfortunately there is a lack of uniform guidelines in terms of what constitutes an adequately clear lumpectomy margin. Substantial debate about bigger margins being better continues [5]. This has led to wide variations in lumpectomy margin reexcision rates from 15 to $47 \%$ [6]. These additional surgical procedures cause significant patient distress, utilize health care resources, and can adversely affect cosmesis. From the patient perspective, they may wonder why we did not get it right the first time. They want their cancer gone while maintaining a normal appearance.

This special issue highlights the areas of controversy and demonstrates current best practices and emerging novel approaches towards optimal breast conservation approach. The goal is to improve our ability to provide breastconserving approaches for breast cancer while avoiding multiple surgical procedures, minimizing recurrence risk while obtaining excellent cosmesis. We have chosen 6 of 16 submissions to be published in this special issue. Each paper was evaluated by at least two expert reviewers and revised according to review comments.

P. Ananthakrishnan et al. provide an excellent comprehensive review article on all aspects involved in optimizing breast conservation. They include discussion of preoperative breast imaging, lesion localization, impact of tumor biology and systemic therapy, intraoperative lesion identification and margin assessment techniques, the role of margin ablation and oncoplastic techniques. They also discuss the promise of ductal anatomy mapping toward the goal of validating the "Sick lobe hypothesis" [7,8] which may allow for more accurate identification of breast tissue to be targeted for excision.

R. Emmadi and E. L. Wiley provide an excellent review from the pathology perspective of the different approaches to margin assessment. They explore issues of specimen processing, fixation, cutting techniques, and reporting. They well explain the reasons for the reporting variations between institutions and the need for standardization.

J. L. Baker et al. present a scholarly review of our current understanding of the issue of atypical ductal hyperplasia $(\mathrm{ADH})$ as it relates to surgical margins. They highlight the large interobserver variability among pathologists in differentiating $\mathrm{ADH}$ from low-grade ductal carcinoma in situ (DCIS). The issue of whether $\mathrm{ADH}$ is a precursor lesion to DCIS is explored.

R. J. Rivera et al. report on a 21-site multicenter clinical trial evaluating the performance of the MarginProbe intraoperative device. This device is based on radiofrequency spectroscopy to assess adequacy of lumpectomy margins. They analyzed volume or resection and reexcision rates in the device group versus usual surgical standard of care (SOC). They demonstrate the reexcision rate of $14.1 \%$ in the device group versus $29.9 \%$ with SOC. Increased resection volume was $2.6 \%$ using the device.

M. M. Chang et al. provide a comprehensive overview of oncoplastic breast reduction. This is a complete review of the 
techniques including indication, patient selection, practical pointers, and their experience including a low (3.3\%) rate of margin failure. They stress the importance of a coordinated team approach between breast surgical oncology, plastic surgery, breast imaging, and radiation oncology.

Lastly, G. H. T. Au et al. present an exciting research paper on margin assessment using a Quantum-Dot Molecular probe in a mouse model. This employs nanoparticle monoclonal antibodies with molecular imaging. Their concept has a potential advantage over optical imaging and radiofrequency spectroscopy in that it is not affected by tissue heterogeneity. It also can display and differentiate very small (100-200 cells) spots. Timeline of 30 minutes is practical for intraoperative use. This early work is an highly innovative approach to a practical issue.

These papers present a great deal of important information and well explore the current state of the art, controversies and future directions towards the important goal of optimizing breast conservation with particular attention to margin issues.

\section{Acknowledgments}

My deepest appreciation to my coguest editors Drs. Ananthakrishnan, Crowe, Dixon, and Fukuma; the authors, reviewers, and the leadership and staff of the International Journal of Surgical Oncology for all their efforts and support to make this special issue a reality.

Sheldon Marc Feldman
[7] T. Tot, "The theory of the sick breast lobe and the possible consequences," International Journal of Surgical Pathology, vol. 15, no. 4, pp. 369-375, 2007.

[8] W. C. Dooley, "Breast ductoscopy and the evolution of the intraductal approach to breast cancer," The Breast Journal, vol. 15, supplement 1, pp. S90-S94, 2009.

\section{References}

[1] B. Fisher, S. Anderson, J. Bryant et al., "Twenty-year followup of a randomized trial comparing total mastectomy, lumpectomy, and lumpectomy plus irradiation for the treatment of invasive breast cancer," The New England Journal of Medicine, vol. 347 , no. 16, pp. 1233-1241, 2002.

[2] N. L. Simone, T. Dan, J. Shih et al., "Twenty-five year results of the National Cancer Institute randomized breast conservation trial," Breast Cancer Research and Treatment, vol. 132, no. 1, pp. 197-203, 2012.

[3] U. Veronesi, N. Cascinelli, L. Mariani et al., "Twenty-year follow-up of a randomized study comparing breast-conserving surgery with radical mastectomy for early breast cancer," The New England Journal of Medicine, vol. 347, no. 16, pp. 1227-1232, 2002.

[4] S. E. Singletary, "Surgical margins in patients with earlystage breast cancer treated with breast conservation therapy," American Journal of Surgery, vol. 184, no. 5, pp. 383-393, 2002.

[5] M. Morrow, J. R. Harris, and S. J. Schnitt, "Surgical margins in lumpectomy for breast cancer, bigger is not better," The New England Journal of Medicine, vol. 367, pp. 79-82, 2012.

[6] P. J. Lovrics, S. D. Cornacchi, F. Farrokhyar et al., "The relationship between surgical factors and margin status after breastconservation surgery for early stage breast cancer," American Journal of Surgery, vol. 197, no. 6, pp. 740-746, 2009. 


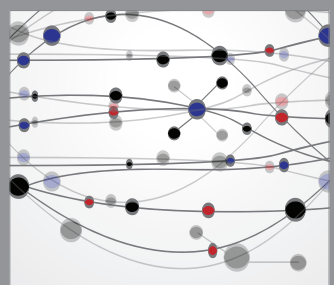

The Scientific World Journal
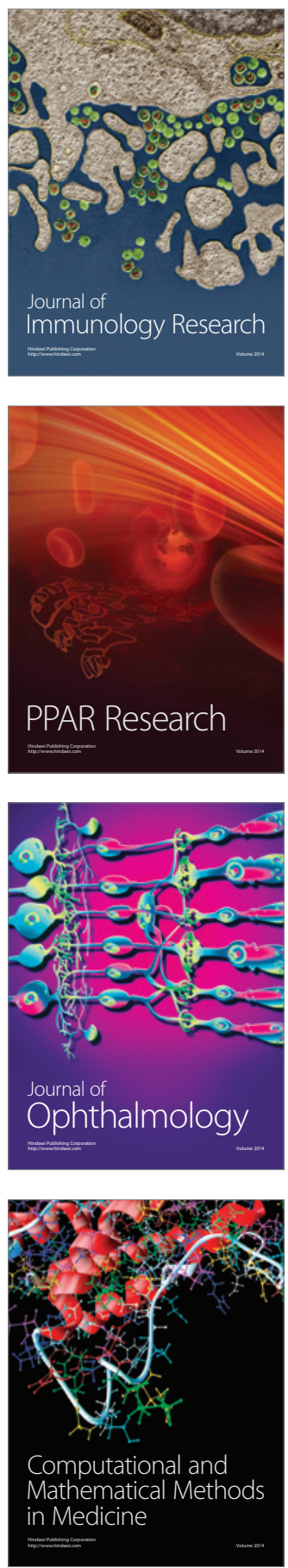

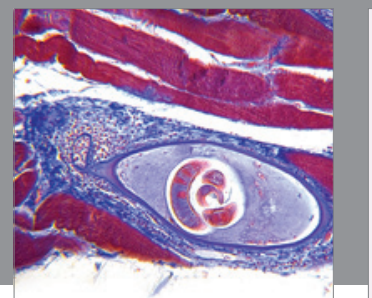

Gastroenterology

Research and Practice
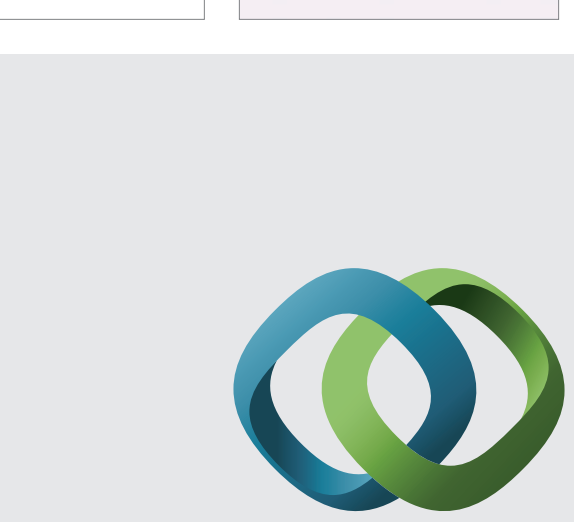

\section{Hindawi}

Submit your manuscripts at

http://www.hindawi.com
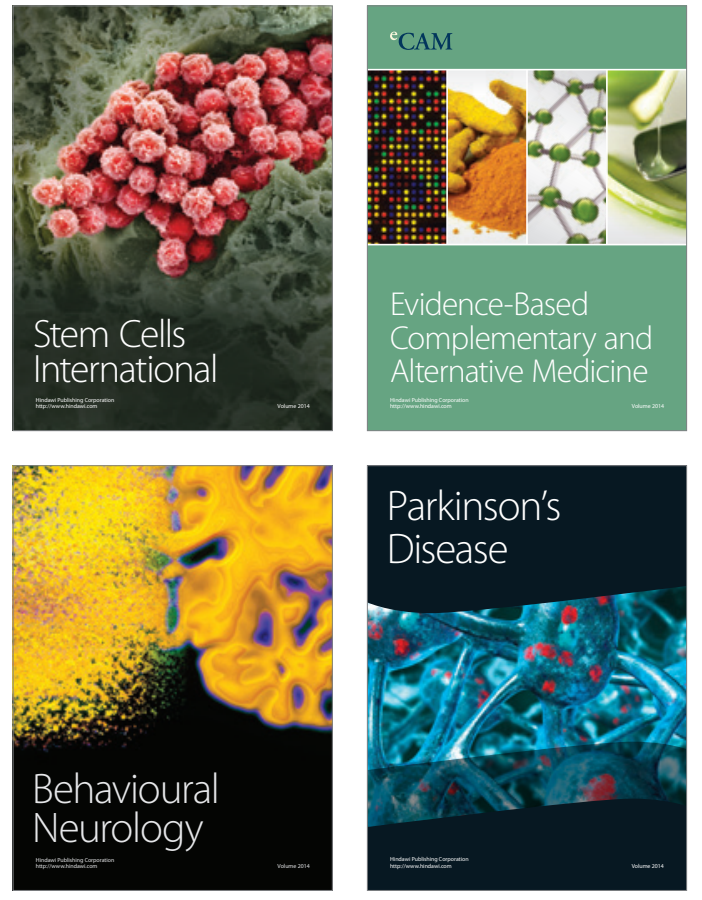
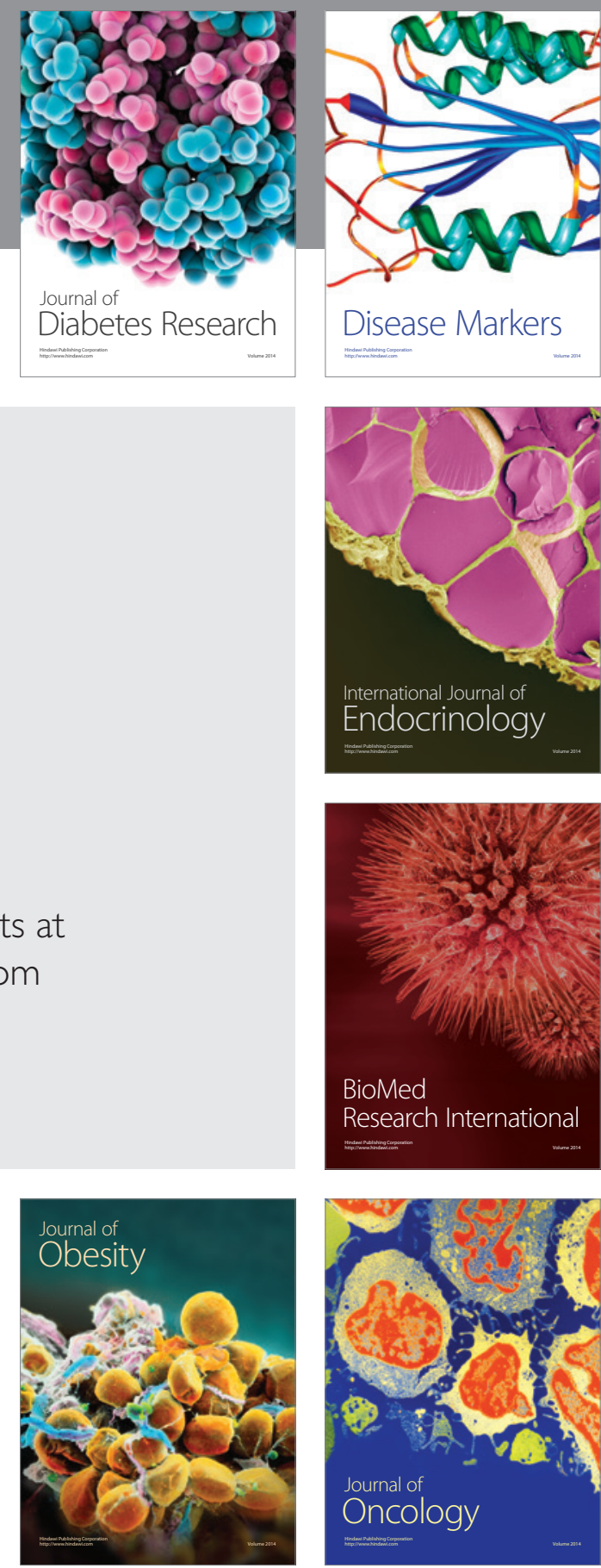

Disease Markers
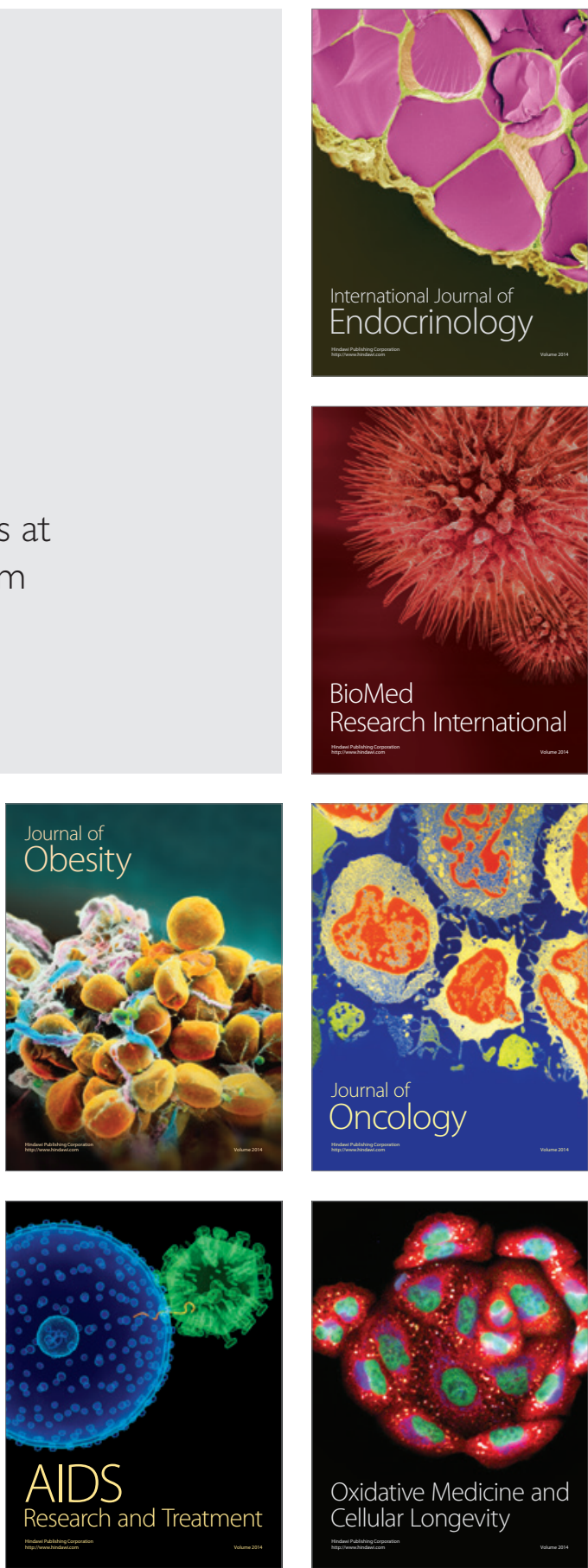